\title{
Gasto farmacéutico derivado de la prescripción de antibióticos a la población pediátrica de Castilla y León en los últimos diez años
}

\author{
ME.Vázquez Fernández ${ }^{\mathrm{a}}$,JM. Eiros Bouzab ${ }^{\mathrm{b}}$ MJ.Vázquez Fernández, F. Martín Pelayod, \\ RM. Bachiller Luque $e^{e}$ C. García de la Ribera ${ }^{f}$ \\ ${ }^{\circ} \mathrm{CS}$ Arturo Eyries (Área Oeste).Valladolid. España. \\ •Área de Microbiología, Facultad de Medicina de Valladolid.Valladolid. España. \\ 'CS. Madrid. España. \\ ¿Subdirector de Redes de la Universidad de Valladolid.Valladolid. España.

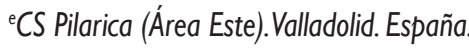 \\ fCS Peñafiel (Área Este).Valladolid. España.
}

Resumen

Introducción: la crisis económica y sus consecuencias están suponiendo dificultades para la sostenibilidad de la prestación farmacéutica y del propio sistema sanitario. Entre los grupos terapéuticos de mayor consumo en población infantil se encuentran los antibióticos.

Objetivo: descripción y análisis del coste del tratamiento en antibióticos prescritos a la población pediátrica de Castilla y León en la última década, en el ámbito extrahospitalario.

Material y métodos: las bases de datos de gasto de antimicrobianos con cargo al Sistema Nacional de Salud provienen de Concylia. Indicadores de consumo: Euros (€)/dosis definida/día y $€ / 1000$ habitantes/día.

Resultados: se ha registrado un gasto en antibióticos de 15750 829,26 €. Las penicilinas asociadas a inhibidores de la betalactamasa (amoxicilina con clavulánico) son responsables del 32,62\% del gasto, seguidas de las cefalosporinas y los macrólidos. En último lugar se encontrarían las penicilinas de amplio espectro (amoxicilina), pese a ser las más prescritas. La evolución interanual refleja un Ilamativo descenso en el precio de la mayoría de los antibióticos, principalmente durante los últimos cinco años. El análisis del gasto disgregado por áreas de salud muestra también importantes diferencias.

Conclusión: las variaciones en el gasto están motivadas principalmente por la frecuencia de uso y por modificaciones en los precios de venta al público. La amoxicilina-clavulánico es el antibiótico que supone más gasto. Los macrólidos son los antibióticos de mayor precio y la amoxicilina el de menor. Se observa una tendencia descendente del gasto en la mayoría de los antibióticos a lo largo del decenio.

Palabras clave: Antibióticos. Gasto sanitario. Población infantil.

Marta Esther Vázquez Fernández, marvazfer@hotmail.com

Este trabajo ha sido financiado por la Gerencia Regional de Salud de Castilla y León. Incluido en los Proyectos en Biomedicina, Biotecnología y Ciencias de la Salud a desarrollar en las Gerencias de Atención Primaria. Resolución 17/07/2010. Ref.: GRS587/B/10. 
Pharmaceutical expenditure derived from antibiotic prescription to the pediatric population in Castilla y León in the last ten years

Abstract

Introduction: the economic crisis and its consequences are posing difficulties for the sustainability of providing a pharmaceutical and health care system. Antibiotics are the therapeutic groups with the highest consumption in children.

Objective: description and analysis of the cost of prescribed antibiotics in the paediatric population of Castilla and Leon in the last decade, in the community setting.

Methods: the databases of antimicrobials' expenditure financed by the NHS come from Concylia. Consume indicators: Euro (€)/DDD and €/1000 inhabitants/day.

Results: there has been an antibiotic cost of 15,750,829.26. Penicillins associated with beta lactamase inhibitors (amoxicillin with clavulanic acid) are responsible for $32.62 \%$ of spending, followed by cephalosporins and macrolides. In the last place are the broad-spectrum penicillins (amoxicillin), although they are the most prescribed antibiotics. The annual evolution reflects a sharp drop in the price of most antibiotics mainly during the last five years. Disaggregated analysis of spending by Health Areas also shows important differences.

Conclusions: variations in spending are driven primarily by the frequency of use and changes in retail prices. Amoxicillin clavulanate is the antibiotic responsible for the highest expense. Macrolides are the most expensive antibiotics and amoxicillin the lowest ones. There is a downward trend in spending in most antibiotics along the decade.

Key words: Antibiotics. Health expenditure. Childhood.

\section{Introducción}

La limitación de los recursos económicos existentes es cada vez más notoria y patente en nuestro Sistema Nacional de Salud (SNS). La crisis económica y sus consecuencias están suponiendo dificultades para la sostenibilidad de la prestación farmacéutica y del propio sistema sanitario ${ }^{1}$. El gasto farmacéutico representa aproximadamente el 20\% del gasto sanitario en España ${ }^{2}$. Entre los grupos terapéuticos de mayor consumo, especialmente en población infantil, se encuentran los antibióticos que suponen entre el 20 y el $23 \%$ de la prescripción total ${ }^{3}$.
Son numerosas las medidas de control de gasto adoptadas, destinadas al uso racional de los medicamentos. El Real Decreto 2402/2004 estableció una bajada de precios de los medicamentos del $4,2 \%$ en 2004 y de un $2 \%$ más en 2005 mediante la reducción en los márgenes de distribuidores y oficinas de farmacia 4 . Posteriormente, en noviembre de 2004 se presentó el I Plan Estratégico de Política Farmacéutica en el SNS 5 , con el objetivo de garantizar la calidad de la prestación farmacéutica, al tiempo que mejorar la eficiencia en el uso de los recursos económicos destinados a su financiación. Más adelante, se aprobó la 
Ley 29/2006, de garantías y uso racional de los medicamentos y productos sanitarios $^{6}$, que sustituyó a la Ley del Medicamento de 1990. Junto a estas medidas, otras acciones también han colaborado en la rebaja de la factura farmacéutica: acciones de promoción de medicamentos genéricos, intensificación de los programas de uso racional de los medicamentos y la contribución y compromiso de las comunidades autónomas (CCAA) $y$ de los profesionales sanitarios. Nuevas reformas emprendidas recientemente en el Real Decreto Ley $4 / 2010$ de 26 de marzo, de racionalización del gasto farmacéutico ${ }^{7}$, establecen más medidas que permiten la reducción del precio de los medicamentos genéricos (un 25\% de media) mediante una regulación más precisa de los descuentos por pronto pago o por volumen de compras que realicen los distribuidores y la industria a las farmacias. De forma complementaria a estas medidas, en el Real Decreto Ley 8/2010, de 20 de mayo, se adoptan medidas extraordinarias para la reducción del déficit público ${ }^{8}$.

En nuestro trabajo realizamos una descripción y análisis del coste del tratamiento en antibióticos prescritos a la población pediátrica de Castilla y León en la última década (años 2001-2010), en el ámbito extrahospitalario.

\section{Material y métodos}

Nuestro estudio se llevó a cabo en el marco de la Atención Primaria de la comunidad autónoma de Castilla y León. La base de datos de gasto de antimicrobianos con cargo al SNS proviene de Concylia (Sistema de Información de Farmacia en nuestra comunidad). El periodo de estudio incluye el consumo efectuado desde el 1 de enero de 2001 hasta el 31 de diciembre de 2010. El gasto farmacéutico se expresó utilizando dos indicadores. El primero, euros por 1000 habitantes y día (€/1000 habitantes/día), se calculó de la forma siguiente: euros $\times 1000 /$ número de habitantes ×365 días.

El segundo indicador utilizado, fue gasto por dosis definida/día (DDD) (€/ DDD), que se obtuvo dividiendo el gasto en euros entre las DDD utilizadas y permitió cuantificar el aporte de cada variable analizada al gasto global.

Se calculó el gasto anual medio de antibióticos describiendo la tendencia en el gasto anual medio. Se realizó una valoración del coste en función de los distintos subgrupos terapéuticos más destacables, haciendo un análisis paralelo del consumo en DDD. Posteriormente, se calculó estratificado el precio de la DDD durante el periodo de estudio para los subgrupos terapéuticos más destacables. 
Por último, se analizó de forma cuantitativa (€/1000 habitantes/día) el gasto de antimicrobianos por áreas de salud y de forma cualitativa utilizando como indicador el gasto en $€ / D D D$. Los resultados se compararon mediante la prueba de Kruskal-Wallis, considerándose diferencias significativas cuando el valor $p<0,05$.

\section{Resultados}

Se ha registrado un gasto en antibióticos de $15750829,26 €$, lo que supone aproximadamente una media de 23,1 $€ / 1000$ habitantes/día y un coste 1,11 $€ / D D D$ de antibiótico prescrito, durante el periodo 2001-2010 para la población pediátrica estudiada. La evolución del gasto farmacéutico derivado del consumo de este grupo de medicamentos a lo largo del decenio muestra una tendencia descendente con un repunte de gasto en el año 2003 y un gasto mínimo en el año 2009 (figura 1).

De manera global las penicilinas asociadas a inhibidores de la betalactamasa (principalmente amoxicilina con clavulánico) son responsables del 32,62\% del gasto, seguida de cefalosporinas y macrólidos (figura 2). En último lugar se encontrarían las penicilinas de amplio espectro (principalmente amoxicilina), pese a ser el más prescrito (tabla 1).

Cuando consideramos la evolución interanual del precio de los grupos te-

Figura 1. Evolución anual del gasto en antibióticos en euros por 1000 habitantes y día en la población pediátrica de Castilla y León.

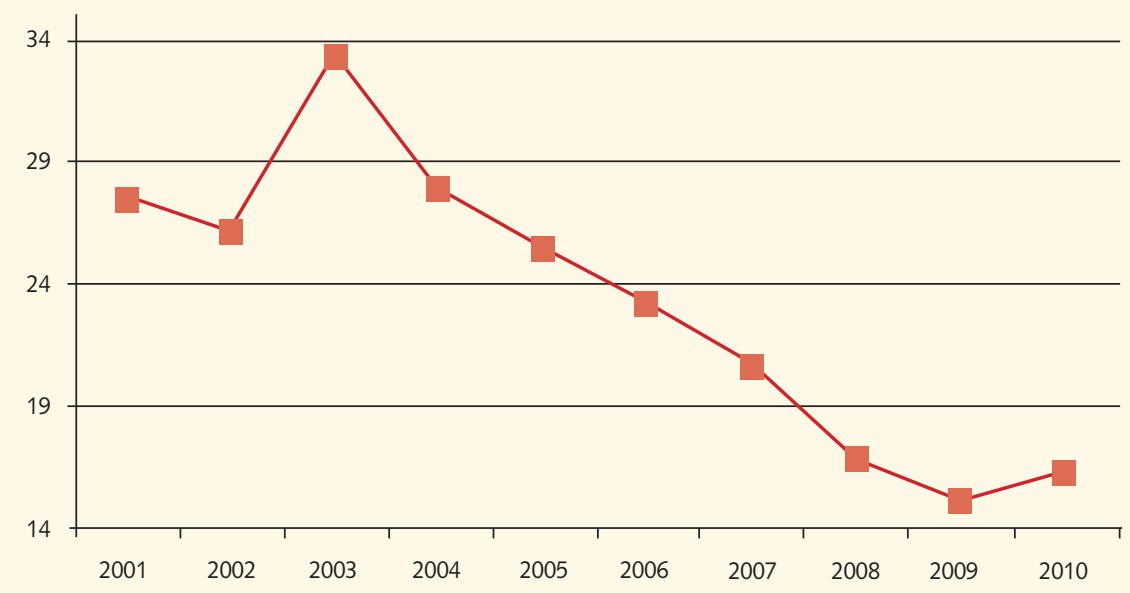


Figura 2. Gasto medio porcentual por subgrupos de antibióticos en euros por 1000 habitantes y día en la población pediátrica de Castilla y León.

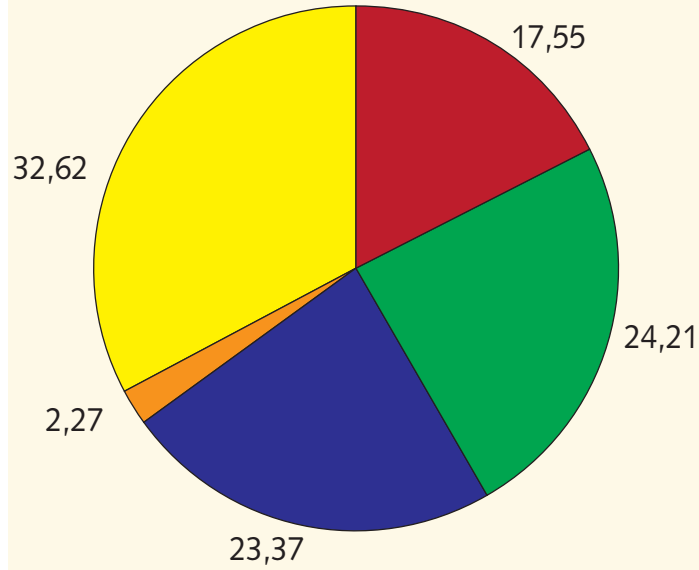

Penicilinas asociadas a inhibidores de la betalactamasa

Penicilinas de amplio espectro Cefalosporinas Macrólidos y lincosamidas Otros

rapéuticos en €/DDD (figura 3 ) obser- péutico, destacando el precio de los mavamos dos hechos llamativos: en primer crólidos, en especial de la azitromicina, y lugar una gran diferencia de coste de la en segundo lugar el llamativo descenso DDD dependiendo del subgrupo tera- del coste de los macrólidos, las cefalos-

\begin{tabular}{|c|c|c|c|c|}
\hline Grupo & Euros & DDD & $\%$ Gasto & $\%$ DDD \\
\hline Cefalosporinas & 3812797 & 1574931 & 24,21 & 11,14 \\
\hline Macrólidos y lincosamidas & 3680177 & 1462138 & 23,37 & 10,34 \\
\hline Resto de subgrupos & 357001 & 309750 & 2,27 & 2,19 \\
\hline $\begin{array}{l}\text { Penicilinas asociadas a inhibidores } \\
\text { de la betalactamasa }\end{array}$ & 5137263 & 4792659 & 32,62 & 33,90 \\
\hline Penicilinas de amplio espectro & 2763482 & 5999480 & 17,55 & 42,43 \\
\hline Totales & 15750720 & 14138957 & 100,00 & 100,00 \\
\hline
\end{tabular}


Figura 3. Evolución anual del gasto en € por dosis definida/día de los subgrupos antimicrobianos en la población pediátrica de Castilla y León.

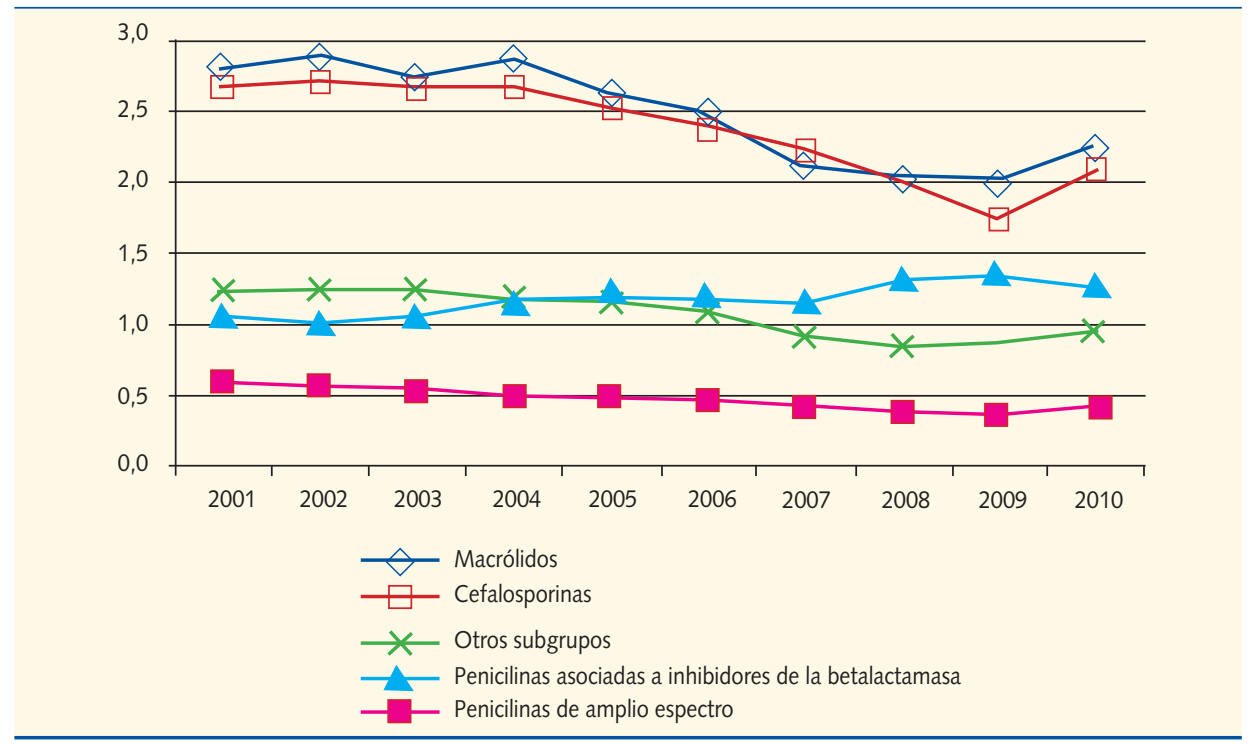

porinas y la amoxicilina-clavulánico $y_{1}$ Bierzo fueron las zonas (gerencias) que en menor medida, de la amoxicilina, presentaron un mayor gasto por antien los últimos cinco años del estudio. bióticos de uso sistémico, entre 30 y 35 El resto de grupos terapéuticos (penici- $€ / 1000$ habitante/día. Las gerencias con linas sensibles a la betalactamasa -fe- menor gasto fueron Salamanca y espenoximetilpenicilina-, penicilinas resis- cialmente Segovia. La disparidad en el tentes a la betalactamasa -cloxacilina-, gasto nos llevó a considerar en cada geaminoglucósidos, sulfamidas, tetracicli- rencia el precio de cada DDD (figura 5), nas, quinolonas y otros), por una par- observando que Salamanca y especialte menos utilizados, experimentan una mente Segovia están utilizando antibiótiligera subida.

El análisis del gasto farmacéutico dis- consumida, es decir que usan los princigregado en áreas de salud y reflejado en pios activos más baratos, mientras Soria la figura 4 muestra diferencias estadísti- y Zamora los de mayor coste, alcanzando camente significativas. Zamora, León y El aproximadamente 1,35€/DDD. 
Figura 4. Gasto global por consumo de antibióticos de uso sistémico en euros por 1000 habitantes y día en cada gerencia.

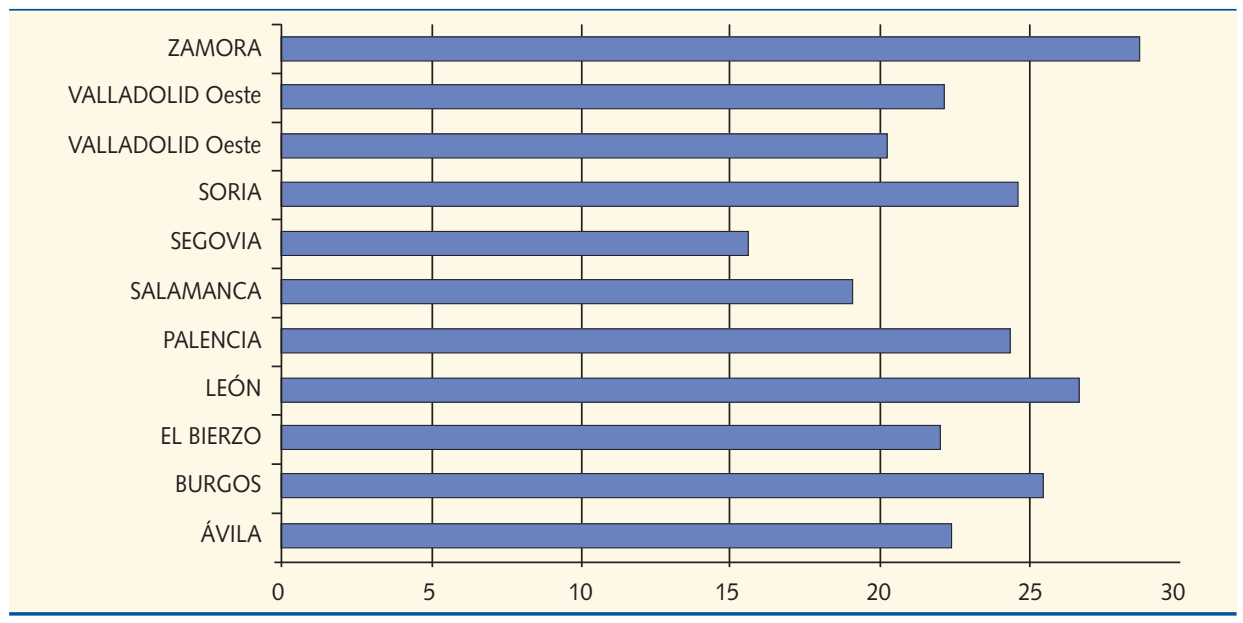

\section{Discusión}

La medida del gasto económico en medicamentos ha sido utilizada desde hace tiempo, sobre todo por los sistemas públicos de atención a la salud, como indicador para la elaboración de presupuestos y contención del gasto ${ }^{9,10}$. La cuantificación del consumo en términos económicos puede ser útil para considerar la importancia real de los medicamentos en un sistema sanitario en un país determinado, siendo imprescindible racionalizar y priorizar la asignación de los recursos disponibles. En el ámbito de la Atención Primaria, la perspectiva económica está insuficientemente analizada en los estudios de utilización de medicamentos. En nuestro trabajo realizamos un análisis de los costes directos derivados del tratamiento antibiótico prescrito a la población pediátrica de Castilla y León y se hace referencia a la prescripción obtenida del correspondiente análisis en DDD como indicador.

Las variaciones del gasto observadas pueden estar originadas por varios motivos: variación en la frecuencia de uso de los distintos antibióticos y modificaciones del precio de venta al público (PVP) a lo largo del periodo. En este sentido, como hemos indicado anteriormente, son numerosas las medidas de contención de precios puestas en marcha por el Ministerio de Sanidad, Política Social e Igualdad ${ }^{4-8}$. Nuestro análisis confirma una curva descendente de gasto a lo lar- 
Figura 5. Diferencias en el gasto medio de antibióticos en cada gerencia, medido en euros por dosis diaria definida.

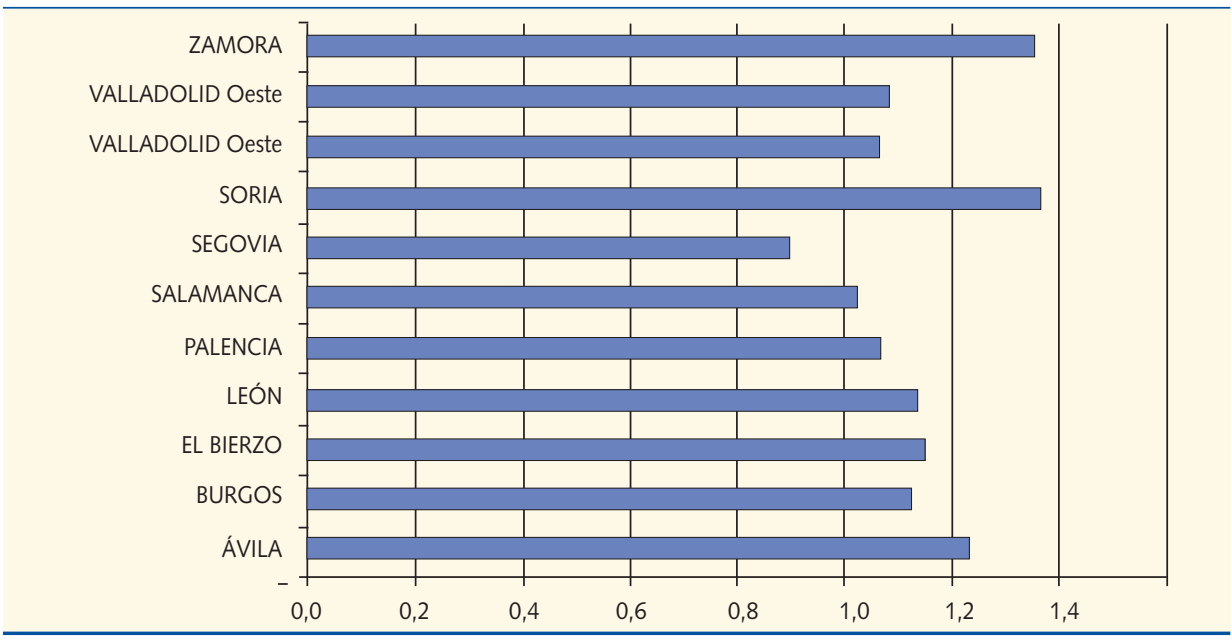

go del decenio, acorde con la caída de precios de los antibióticos en el mercado, tan solo rota en el año 2003 por el mayor consumo de antibióticos y con un mínimo en los tres últimos años, en los que parece que hemos tocado fondo.

El primer punto importante valorado es la distribución del gasto por subgrupos, observando cómo las penicilinas de amplio espectro que supusieron el $42,43 \%$ en DDD consumidas originaron tan solo el $17,55 \%$ del gasto. Encabezando la lista de subgrupos que ocasionan mayor gasto se situarían las penicilinas asociadas a inhibidores de la betalactamasa, principalmente la amoxicilina-clavulánico. Por otro lado, al comparar el comportamiento interanual, a partir del año
2005 observamos una tendencia descendente del gasto para los principales subgrupos terapéuticos, especialmente para los macrólidos y las cefalosporinas, que experimentan una evolución bastante paralela que confirma la importante caída de precios y utilización. El dominio en el perfil de gasto de nuestro estudio de las penicilinas asociadas a inhibidores de la betalactamasa concuerda con los resultados obtenidos años antes en la población general por Pastor y cols. ${ }^{11}$ en la provincia de Valladolid, y por Castán y cols. $^{12}$, en la provincia de Zaragoza. Sobre el resto de subgrupos (especialmente los que engloban a fenoximetilpenicilina, trimetropim-sulfametoxazol y cloxacilina), no observamos el efecto de las me- 
didas de contención emprendidas políticamente, ya que la tendencia observada es a elevar discretamente su precio en el mercado. Sin embargo, debido al escaso volumen de DDD prescritas en la población pediátrica analizada, su repercusión en el gasto final es baja.

La variabilidad del gasto encontrada en las distintas áreas de salud de nuestra comunidad traduce las diferencias cuantitativas de prescripción en DDD y las diferencias cualitativas en el precio de los distintos principios activos utilizados. No influyó en las diferencias la modificación de los precios en el mercado, ya que en todos los entornos se aplican las mismas tarifas a lo largo del periodo. Así, encontramos que la gerencia con una mejor relación de gasto por DDD fue Segovia, situándose en el último lugar del gasto farmacéutico, mientras que Zamora fue una de las gerencias con mayor gasto, debido principalmente a la utilización de antibióticos de mayor precio por DDD utilizada. Aunque en términos generales no encontramos las causas de tanta variabilidad, suponemos que se debe a la intervención de diversos factores, como son la relación médicopaciente, factores epidemiológicos, estilos personales de prescripción, etc., comentados en otros artículos ${ }^{13}$.

Este trabajo sigue la línea del realizado por Pastor y cols. ${ }^{11}$ en el periodo 1996-
2000. Estos autores encontraron un gasto por DDD que osciló entre 1,69€ de máximo y $1,55 €$ de mínimo, para la población general de la provincia de Valladolid, confirmando nuestros mejores resultados de 1,11 €/DDD en la población pediátrica de Castilla y León. Estos datos confirman el menor precio de los antibióticos en momentos más actuales y el menor gasto por DDD en la población pediátrica, hecho este último que atribuimos principalmente a una mayor concienciación del profesional y a una mejor adaptación a las guías terapéuticas que indican la prescripción de penicilinas de amplio espectro para las infecciones respiratorias no complicadas del niño ${ }^{14}$.

\section{Conclusiones}

- Se observa una tendencia descendente del gasto en la mayoría de los antibióticos a lo largo del decenio, relacionada principalmente con dos motivos: la caída de los PVP y la disminución de la frecuencia de consumo.

- Las penicilinas asociadas a inhibidores de la betalactamasa, principalmente amoxicilina-clavulánico, son los antibióticos que suponen más gasto.

- Los macrólidos son los antibióticos de mayor precio y la penicilina de 
amplio espectro, amoxicilina, el de menor.

- La variabilidad de gasto presentado en las distintas áreas de salud confirma el patrón heterogéneo de prescripción de los diversos profesionales influidos por distintos factores.

\section{Bibliografía}

1. Sánchez Bayle M, Palomo Cobos L. Coste y sostenibilidad del Sistema Sanitario Publico español. Tribuna Libre. 2011;197:12-15 [en línea] [consultado el 15/05/2011]. Disponible en http://atencionprimaria.org.es/sites/default/fi les/documentos/667-Abr-2011/img025.pdf

2. Strategic Research Center. El gasto farmacéutico en España. EAE Bussiness School; 2010 [en línea] [consultado el 15/05/2011]. Disponible en http://heraldodeoregon.files.wordpress. com/2011/04/el-gasto-farmaceutico-en-espac 3b1a-eae-busines-school.pdf

3. Clavenna A, Berti A, Gualandi L, Rossi E, De Rosa $M$, Bonati $M$. Drug utilisation profile in the Italian paediatric population. Eur J Pediatr. 2009;168:173-80.

4. Real Decreto 2402/2004, de 30 de diciembre, por el que se desarrolla el artículo 104 de la Ley 25/1990, de 20 de diciembre, del Medicamento, para las revisiones coyunturales de precios de especialidades farmacéuticas y se adoptan medidas adicionales para la contención del gasto farmacéutico [en línea] [consultado el 08/05/2011]. Disponible en www.boe.es/boe/ dias/2004/12/31/pdfs/A42819-42905.pdf

5. Rodríguez Sendin JJ, Toquero F, FernándezPro A, Fumadó J, Fernández J, Hidalgo P y cols. Plan Estratégico de Política Farmacéutica para el Sistema Nacional de Salud [en línea] [consultado el 15/05/2011]. Disponible en www.semg.es/ doc/documentos_SEMG/informe_PEPF.pdf
6. Ley $29 / 2006$, de 26 de julio, de garantías y uso racional de los medicamentos y productos sanitarios [en línea] [consultado el 15/05/20111]. Disponible en www.boe.es/aeboe/consultas/ba ses_datos/doc.php?id=BOE-A-2006-13554

7. Real Decreto-ley 4/2010, de 26 de marzo, de racionalización del gasto farmacéutico con cargo al Sistema Nacional de Salud [en línea] [consultado el 15/05/2011]. Disponible en www.boe.es/boe/ dias/2010/03/27/pdfs/BOE-A-2010-5030.pdf

8. Real Decreto-ley 8/2010, de 20 de mayo, por el que se adoptan medidas extraordinarias para la reducción del déficit público [en línea] [consultado el 15/05/2011]. Disponible en www.boe.es/boe/dias/2010/05/24/pdfs/BOEA-2010-8228.pdf

9. García-Sempere, Peiró S. Gasto farmacéutico en Atención Primaria: variables asociadas y asignación de presupuestos de farmacia por zonas de salud. Gac Sanit. 2001;15:32-40.

10. Soto J. Estudios de farmacoeconomía: ¿por qué, cómo, cuándo y para qué? Medifam. 2001;11:147-55.

11. Pastor García E. Estudio farmacoeconómico del consumo de antibióticos de uso sistémico en la provincia de Valladolid. Periodo 1996-2000. Tesis doctoral. Facultad de Medicina de Valladolid; 2001.

12. Castán Cameo S, García Latorre FJ, Martínez Gorostiaga J, Sierra Moros MJ, Solano Bernad VM, Peral Casado A. Un estudio de minimización de costes en la prescripción de antiinfecciosos en 
dos áreas de Atención Primaria. Rev Esp Salud Pública. 1998;72:33-42.

13. Vázquez Fernández $M E$, Pastor García $E$, Bachiller Luque MR, Vázquez Fernández MJ, Eiros Bouza JM. Análisis del gasto derivado de la prescripción de antibióticos en la población pediátri- ca de Castilla y León. Rev Pediatr Aten Primaria. 2008;10:55-66.

14. Consejería de Sanidad. Gerencia Regional de Salud. Guía terapéutica Sacyl. Problemas de salud prevalentes en Pediatría. Valladolid: Junta de Castilla y León; 2008.

\section{खृ్య}


